\title{
A Shunt Active Power filter based on Instantaneous Reactive Power Compensation and Direct Voltage Control for Medium-Voltage Applications
}

\author{
V. Krishnaveni ${ }^{1 *}$, C. pavan kalyan ${ }^{2}$ \\ ${ }^{* 1}$ M. Tech student, ${ }^{2}$ Assistant professor, Electrical and Electronic Department, \\ ${ }^{* 1,2}$ St. Theressa Institute of Engineering and Technology \\ Email: vysyarajukrishna@gmail.com
}

\section{Corresponding Author: -}

V. Krishnaveni,

Email: vysyarajukrishna@gmail.com

\begin{abstract}
: -
high power and medium voltage (MV) applications need Controlled rectifiers such as 12-pulse converters, because of the high reliability, low complexity, robustness and low power losses. But having the drawback of harmonics generation causes power quality problem. Power quality can be improved with passive filters but having disadvantages such as dependency on the source impedances, parallel and series resonance, aging of passive components. Active power filtering $(A P F)$ is used as compensation technique in order to improve the power quality at the line side. Hence APF becomes a promising compensator solution. This paper presents the APF for a Medium-Voltage applications using Open Loop Control Compensation
\end{abstract}

Key words: - 12-Pulse converters, Medium voltage applications, open-loop control, SAPF, power quality mitigation, $P C C$.

\section{(ㄷ) (3) (1)}




\section{INTRODUCTION}

High power and medium voltage (MV) applications need Controlled rectifiers such as 12-pulse converters, because of the high reliability, low complexity, robustness and low power losses. But having the drawback of harmonics generation causes power quality problem. [1]-[3]. The compensating signal is the result of three controlling actions which are current control, reference extraction, and the PWM [3], [6]. When the switching frequency is at low level the delay introduced in the reference signals. In general, these delays are offered by the measuring devices, digital signal processing (DSP) and the current control response. As a result, the THD of compensated current is more than allowed standards. The APF overcomes filter drawbacks and is robust and reliable too [12], [13]. Moreover, APF performance is having drawbacks in MV and high voltage systems due to having limitations in switching frequency [1]. To solve such a problem some solutions have been projected. More than a few conventional APF techniques are used low-pass filters (LPF) to get the current harmonics and classical controllers for the current control such as PI or hysteresis [14]. These consequences harmonic miss-cancellation and phase shifts and errors in reference tracking, as a result degrading the performance of the filtering. To determine individual harmonics a Fourier series has been used [15].

This paper presents control techniques to conquer SAPF delay effects [3] the objective of this paper is to avoid passive elements, have a less calculation burden, and avoid pre- experimental results [16], [20] where, an open- loop technique, with a genuine algorithm, is easy to implement and gives better compensation performance, with low THD introduced. This technique has two stages: preparation and operation.

The paper is organized in five sections. Section II presents the proposed system. Section III introduces the open loop compensation principle and the associated filter current generation and modulating signal determination. Section IV explains how to compensate selected dominant harmonics in the main ac current. Section V presents the performance evaluation for the compensated system.

\section{CURRENT HARMONIC COMPENSATION of SHUNT APF}

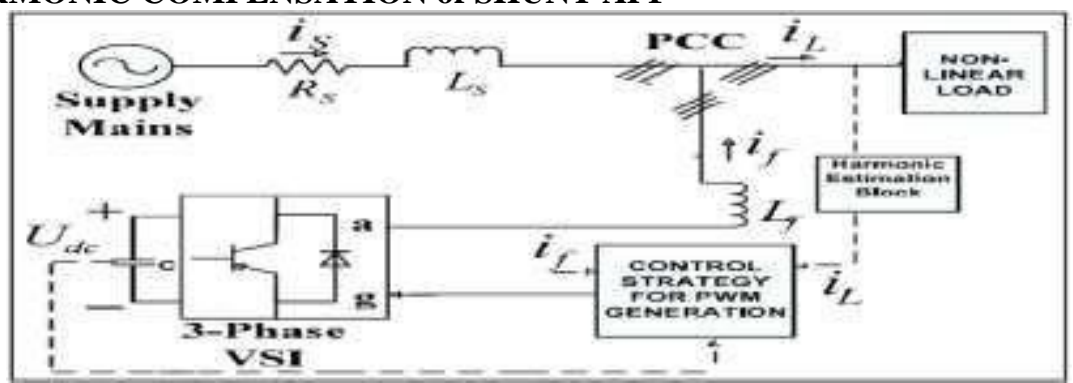

Fig.1 Basic diagram of shunt APF for current harmonic compensation

Basic block diagram of SAPF for current harmonic compensation is depicted in figure 1. Here a voltage source inverter (VSI) shunted with capacitor. The voltage across capacitor is termed as dc-link voltage; the dc-link voltage needs to be constant for proper operation of filter. The filter, non-linear load and the source are connected at point of common coupling (PCC). The firing pulses are provided to the inverter which is generated using the control block. The dc-link voltage, source and filter current are sensed and passed to the control block for generating the firing pulses. Here the basic idea of operation is that when any nonlinear load demands non-linear current from source, it has to supply that current when filter is not used. But when filter scheme like shunt active power filter is being used in the system, it supply the harmonic of the current whereas supply provides the sinusoidal current. In other way we can think of filter is generating

\section{COMPENSATED 12-PULSE CONVERTER}

the system shown in fig. 1 is constructed with a three-winding transformer and one SAPF connected to secondary taps which offers filter side voltage reduction, without a high bandwidth step-down transformer. This auto- transformer type configuration reduces the voltage rating of the SAPF switches, consequently, increases the switching frequency limit. This arrangement mitigates the net amp- turns within the transformer window area for the current harmonic components generated by each 6-pulse converter, with the net harmonics compensated by the SAPF. In the case of symmetrical operation, $\alpha 1=\alpha 2$, the 5 th and 7 th current harmonic components generated by each 6 -pulse converter cancel due to the transformer phase shifting property of the secondary's and the SAPF does not need to inject these harmonic components. In the case of asymmetrical operation, the harmonic components are partially mitigated and the necessary SAPF compensation current is minimized. The SAPF is controlled using predictive current control which has the disadvantage of only one sample time delay in addition to extracting the reference current from the PCC voltage, which may be distorted [3]. 


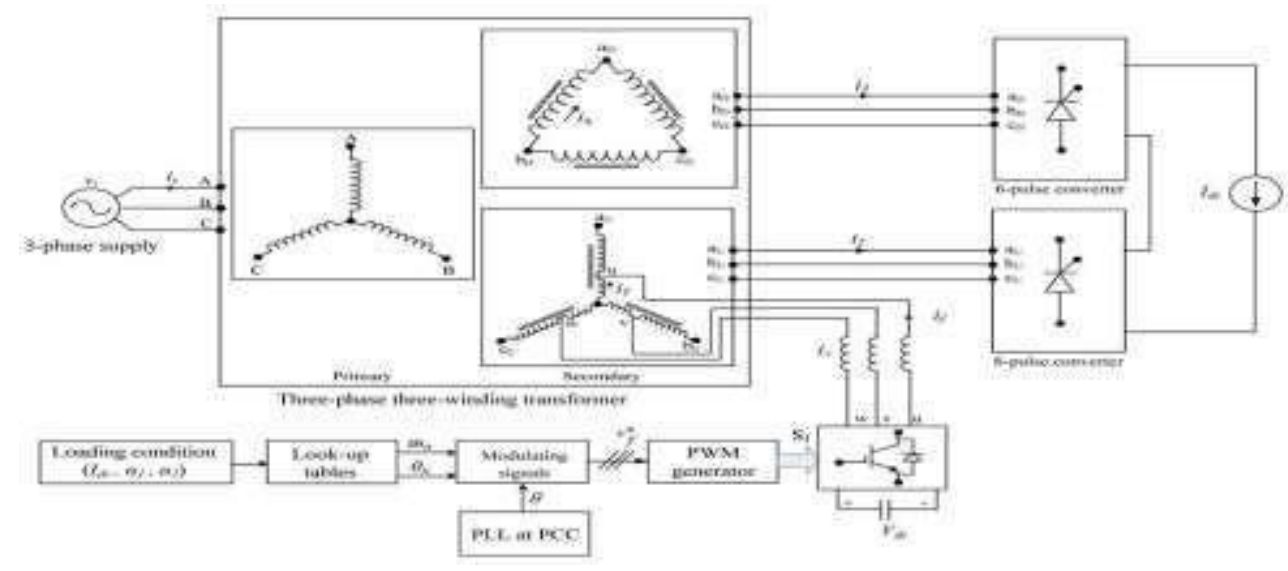

Fig. 2 the system under study, compensated 12-pulse converter using an open-loop controlled APF.

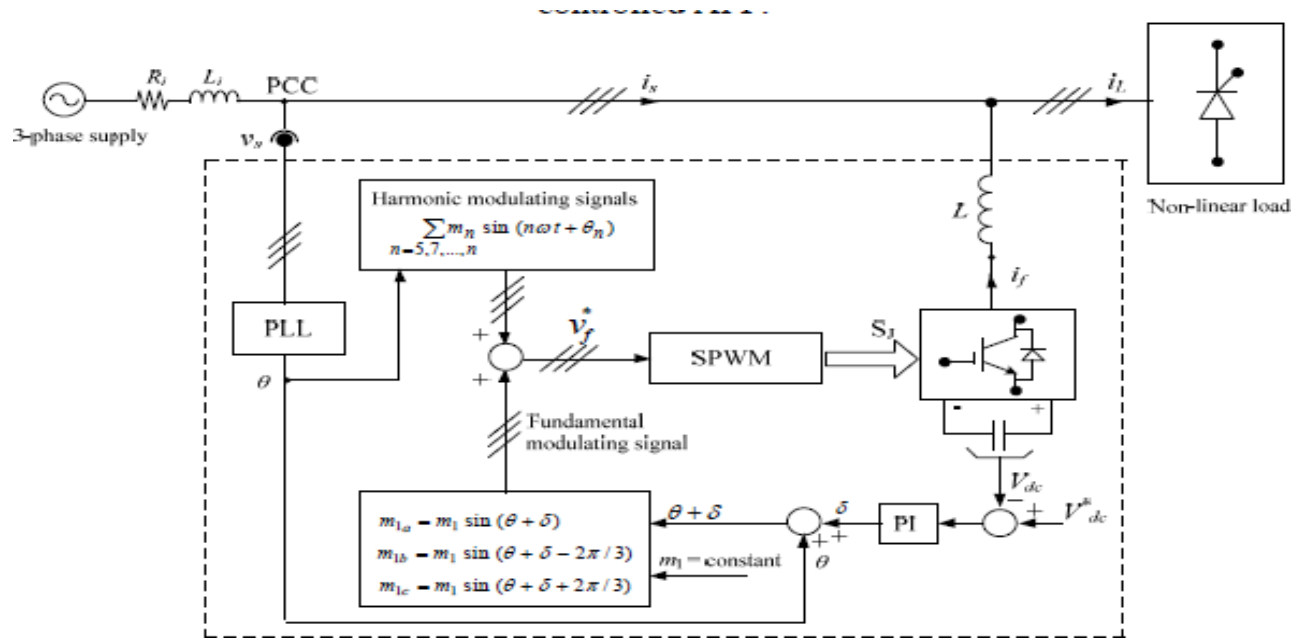

Fig. 3. Basic compensation principle using an open-loop controlled shunt APF.

\section{IV.CONTROLLING TECHNIQUES}

In any active power filter system, control algorithms has major role in deciding the performance of harmonic compensation. The gate pulses provided are provided using the control algorithms to the voltage source inverter used in filtering system. It makes a closed loop control on the harmonic current present in the line and compares with ac sinusoidal source to get the error. This error is passed through some controllers and control algorithms to generate pulses for VSI. The reliability and performance of any active power filtering largely depend on control algorithms adopted, there are number of algorithms proposed in the last decade some of which work good under balanced and unbalanced conditions also. Input to the control block is source current, load current and the DC link voltage. The performance of compensation of harmonics of source current largely depend on the algorithm adopted since the control methods are responsible for generating the reference currents which used to trigger the Voltage Source Inverters (VSI). The APF needs an accurate control algorithm that provides robust performance under source and load unbalances. Better control strategy leads to better dynamic response of the system. Here the control block shown in above figure is responsible for generating pulses for the VSI inverter, with which inverter used to operate and supply filter current. Some of the control strategies are discussed below:

Pulse width modulation techniques: The developments in fully controllable power semiconductors and high speed digital processors have made various pulse width modulation (PWM) techniques applicable to power converters.

PWM techniques present

- cost effective, efficient and more compact systems,

- Nearly sinusoidal input/output waveforms,

- Superior control characteristics.

In the application of PWM techniques to CSC given in Figure must be guaranteed. This constraint implies that there is always at least one switch from the upper half bridge (S1, S3, S5) and at least one switch from the lower half ridge (S2, $\mathrm{S} 4, \mathrm{~S} 6$ ) should be in conduction in order to provide a circulating path for the dc-link current through the supply or the converter.

Different PWM techniques have been studied for CSC. Among these studies, following PWM techniques are mainly used:

- Modified Sinusoidal PWM (MSPWM)

- Space Vector PWM (SVPWM) 
- Selective Harmonic Elimination Method (SHEM).

Figure.4 shows the Control block module for generating gate pulses using SVPWM technique.

Table 1 COMPARISON OF DIFFERENT MODULATION TECHNIQUES

\begin{tabular}{|c|c|c|c|}
\hline & Harmonic spectra & Modulation index & applications \\
\hline MSPWM & $\begin{array}{l}\text { Smaller low order } \\
\text { harmonics for fs }>2 \mathrm{KHZ} \\
\text { Fg=(2/3)f carrier }\end{array}$ & Controllable $0<\mathrm{M}<1.0$ & $\begin{array}{l}\text { Medium power IGBT or } \\
\text { MOSFET } \\
\text { converters, where fased } \\
>2 \mathrm{KHZ}\end{array}$ \\
\hline SVPWM & $\begin{array}{l}\text { Smaller low order } \\
\text { harmonics for fs }<2 \mathrm{KHZ} \\
\mathrm{Fg}=(1 / 3) \text { f carrier }\end{array}$ & Controllable $0<\mathrm{M}<1.0$ & $\begin{array}{l}\text { Medium power IGBT or } \\
\text { MOSFET } \\
\text { converters, where fs } \\
>2 \mathrm{KHZ}\end{array}$ \\
\hline SHEM & $\begin{array}{l}\text { Negligible low order } \\
\text { harmonics }\end{array}$ & $\begin{array}{l}\text { Can only be controlled in } \\
\text { discrete steps }\end{array}$ & $\begin{array}{l}\text { Medium or high power } \\
\text { IGBT or IGCT based } \\
\text { converters } \quad \text { where } \\
\text { fs }<1 \mathrm{KHZ}\end{array}$ \\
\hline
\end{tabular}

Where,

Fs= switching frequency

$\mathrm{K}=$ number of harmonics to be eliminated Fcarrier = carrier frequency

$\mathrm{F} 1$ = fundamental frequency

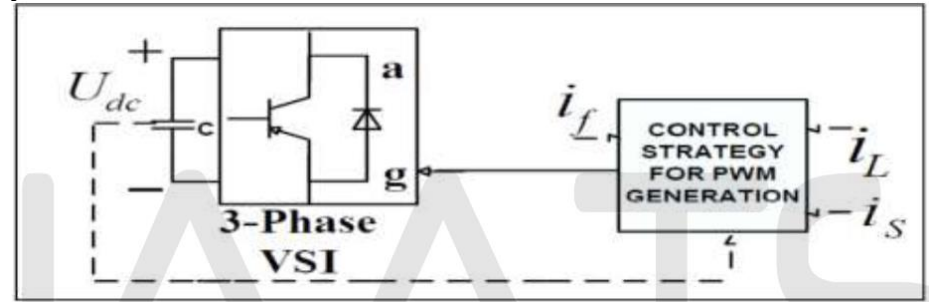

Figure.4 Control block module for generating gate pulses

\section{OPEN LOOP COMPENSATION PRINCIPLE}

The column basic compensation principle of the open-loop controlled shunt SAPF for a non-linear load is shown in fig. 2. By knowing the non-linear load operating conditions, the firing delay angle, and the load current which is assumed ripple free, the $n^{\text {th }}$ harmonic current to be injected at the PCC can be calculated. This current cancel the corresponding $n^{\text {th }}$ harmonic supply current. These desired compensating harmonic current vectors can be determined by setting the correct magnitudes and phase-shift angles of the harmonic contents. An PLL is used to synchronize the compensation process. The dc-link capacitor voltage is controlled to achieve power balance between the filter and the mains through a separate control loop. It is controlled at a level to avoid over modulation after adding the harmonic modulating signals. Time shifting the fundamental modulating signal may be required to compensate any system delay and to achieve minimum fundamental filter current [21]. The following subsections explain filter current generation and how to determine the modulating signal.

to avoid over modulation which occurs when the peak of the overall modulating signal represented by equation (1)

$$
v_{f}^{*}(\omega t)=\sum_{n=1,5,7, \ldots} m_{n} \sin \left(n \omega t+\theta_{n}\right)
$$

The Relation between Load Current and Modulating Signals is give by the equation (2)

Where

$$
i_{T}(\omega t)=i_{2}(\omega t)-i_{f}(\omega t)
$$

$i T=$ current flowing between the neutral point and the tapping (injecting) point

$i 2=$ star-connected secondary output current, and

if $=$ is the compensating (filter) current.

$i s$, in terms of the transformer currents and the step-down ratio is

$$
i_{s}(\omega t)=\left(1-a_{1}\right) \frac{N_{2}}{N_{1}} i_{2}(\omega t)+a_{1} \frac{N_{2}}{N_{1}} i_{r}(\omega t)+\frac{N_{3}}{N_{1}} i_{\Delta}(\omega t)
$$

The compensating current is given by the equation (4) 


$$
i_{f}(\omega t)=\frac{1}{a_{t}} \frac{N_{2}}{N_{1}}\left(i_{h}(\omega t)-i_{s}(\omega t)\right)
$$

The compensation performance of the Active filter based on Instantaneous Reactive Power and Direct Voltage Control is investigated for a MV system using MATLAB/SIMULINK software and for conformance; experimental results from a voltage scaled system are presented.

a) Active Filter based on Instantaneous Reactive Power Compensation

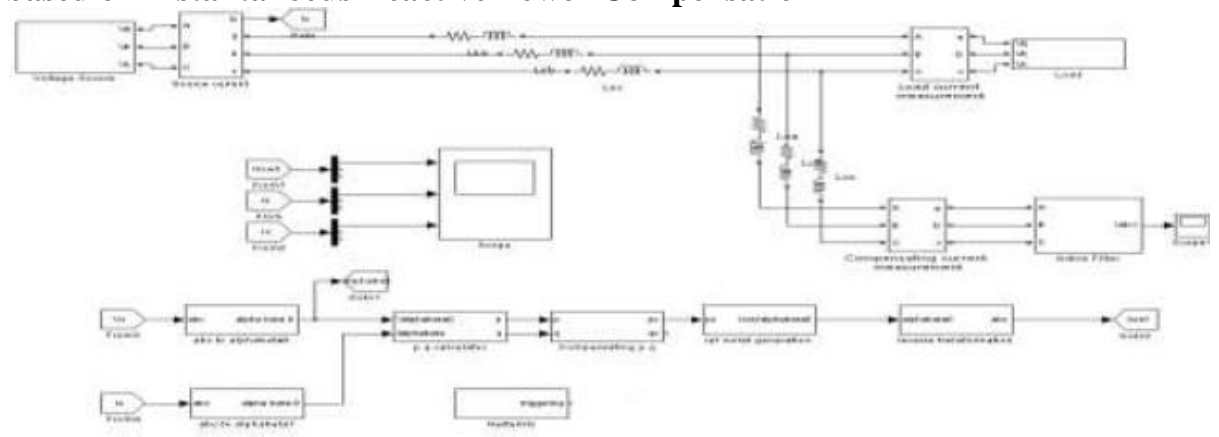

Figure 5. MATLAB/SIMULINK model of Shunt Active Filter based on Instantaneous Reactive Power Compensation

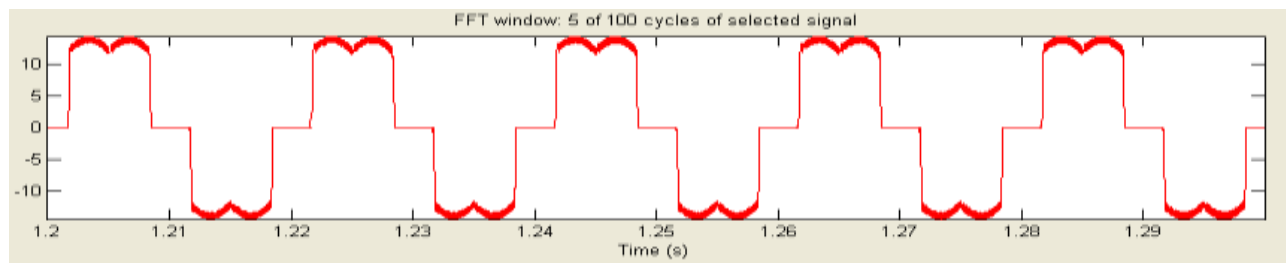

Fig.6 Load current waveform IL

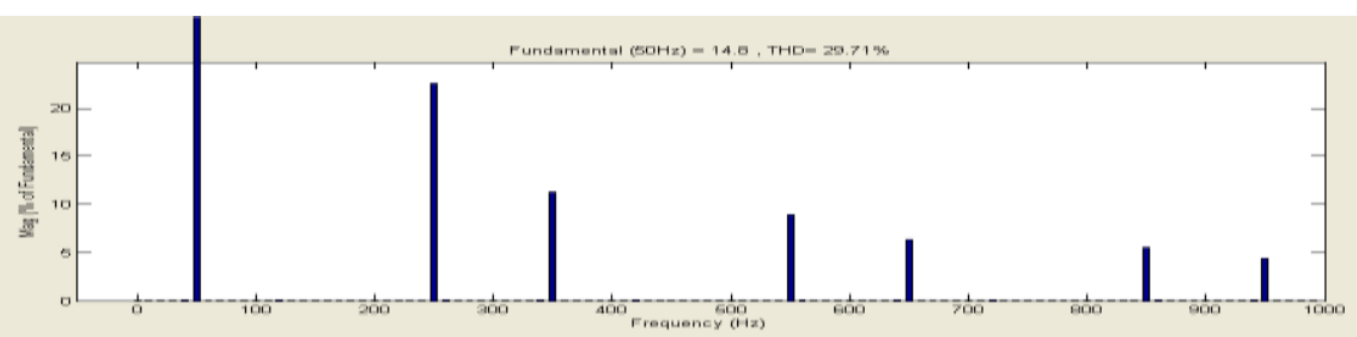

Fig.7 FFT analysis of Load current Spectrum

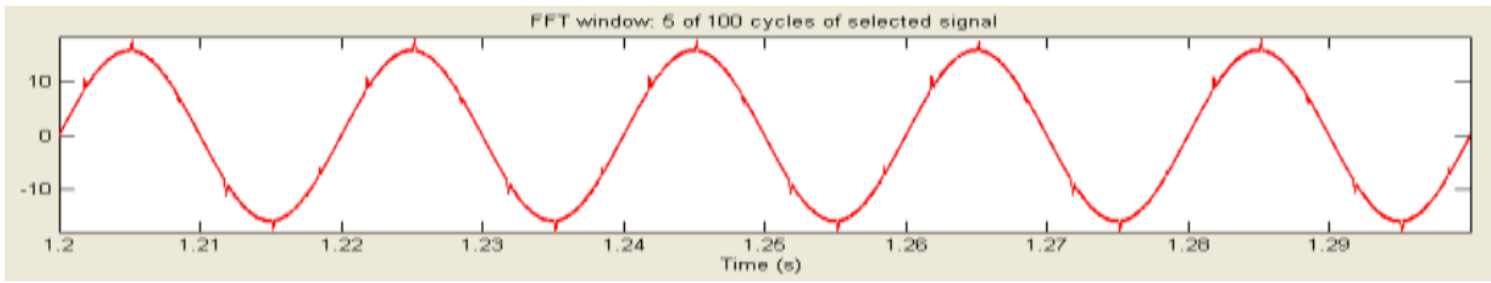

Fig.8 Source Current Waveform IS

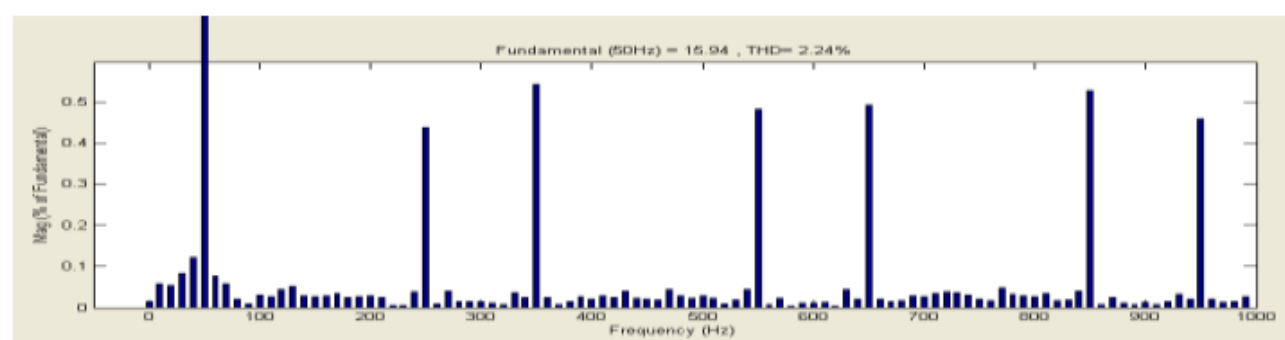

Fig.9 FFT analysis of Source current Spectrum 


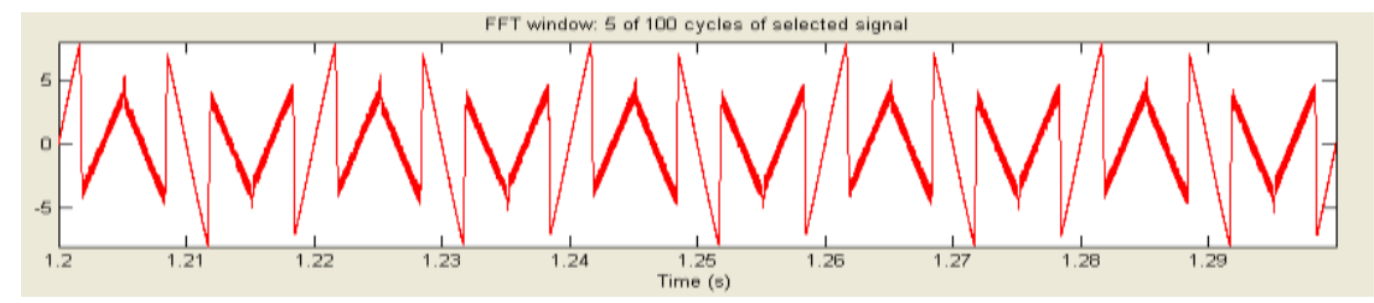

Fig.10 Compensating Current Waveform Ic

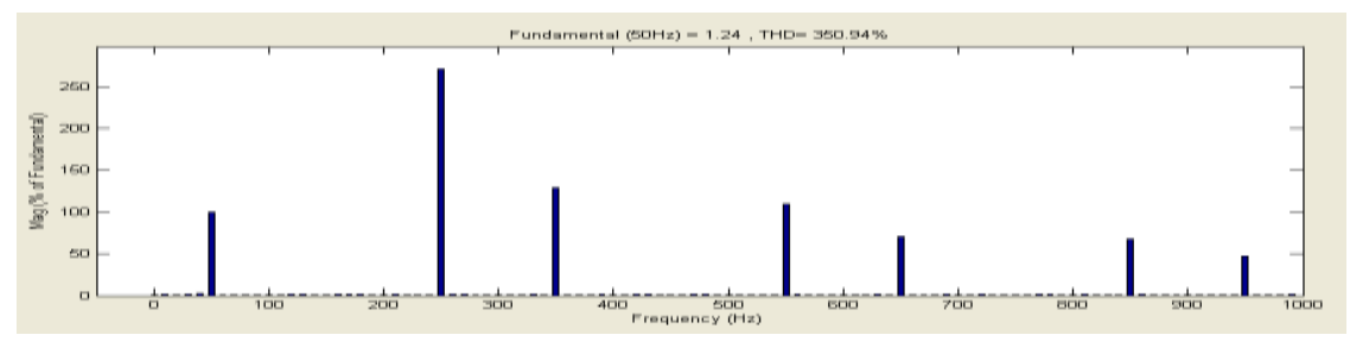

Fig.11 FFT analysis of Compensating current Spectrum

Figure 15 shows the shows the amplitude of various harmonics of Compensating current expressed as a percentage of fundamental. The fundamental magnitude is $1.24 \mathrm{amps}$ and the THD is $350.94 \%$.

\section{Active Filter based on Direct Voltage Control}

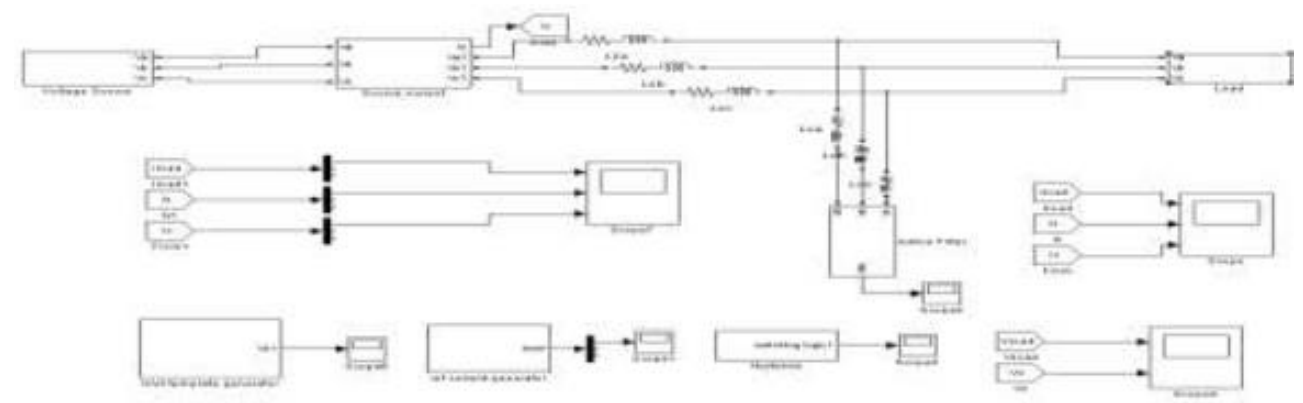

Fig.12 MATLAB/SIMULINK model of Shunt Active Filter based on Direct Voltage Control

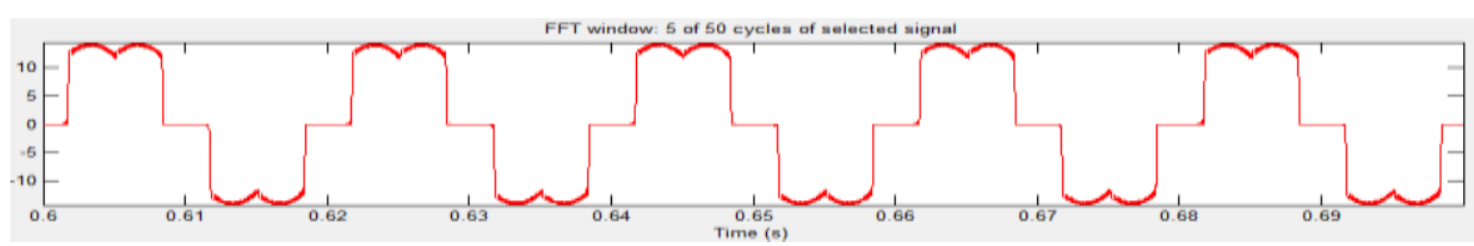

Fig.13 Load current waveform IL

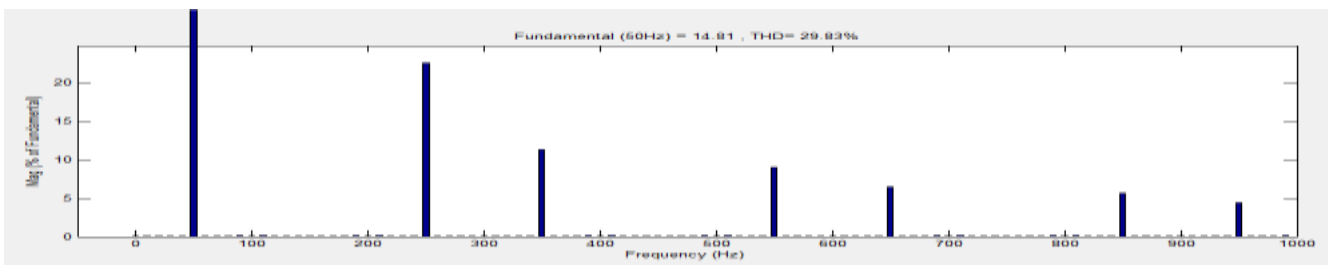

Fig.14 FFT analysis of Load current Spectrum

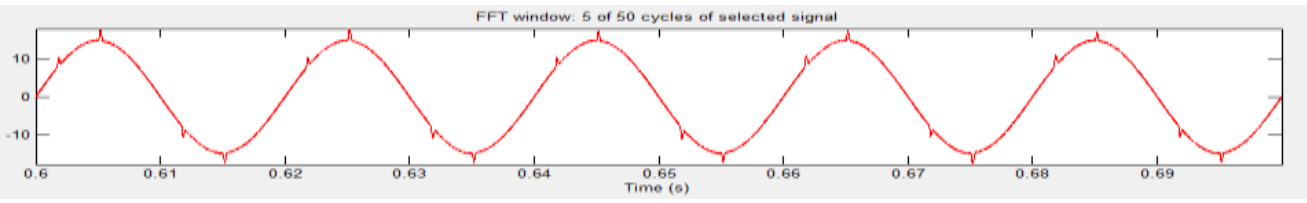

Fig.15 Source Current Waveform IS 


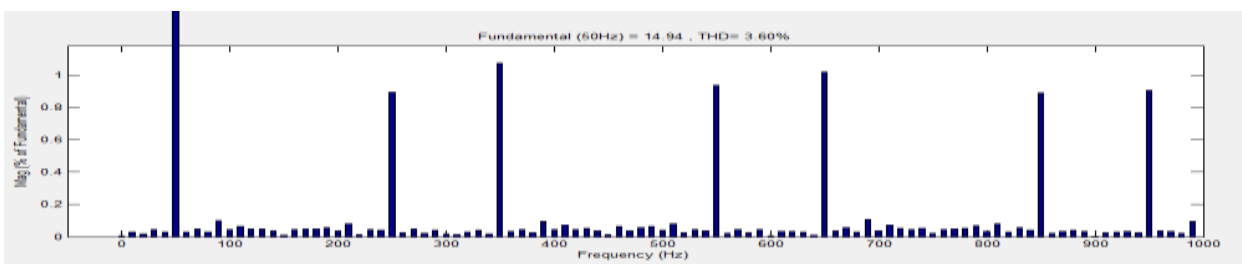

Fig.16 FFT analysis of Source current Spectrum

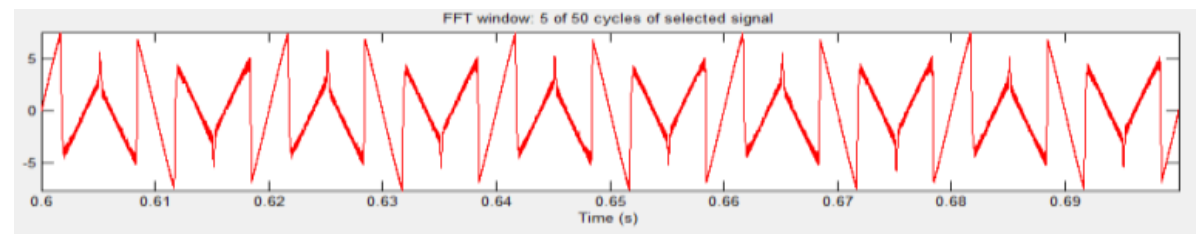

Fig.17 Compensating Current Waveform Ic

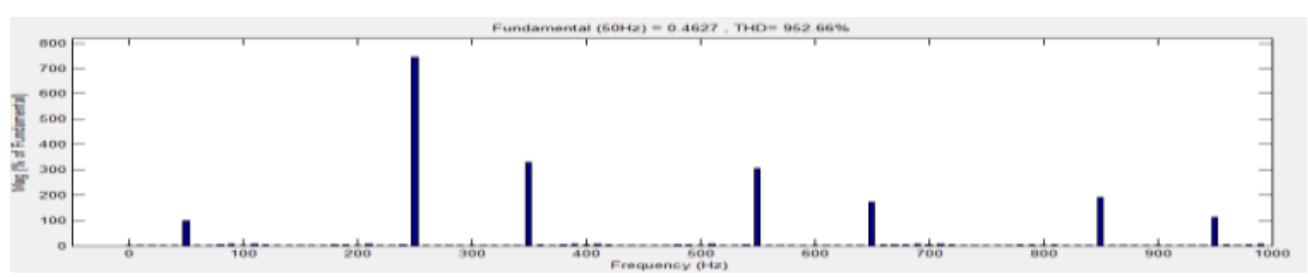

Fig.18 FFT analysis of compensating current Spectrum

\section{CONCLUSION:}

In this paper 12-pulse converter is used to fulfill the requirements for the medium voltage (MV) applications like the high reliability, low complexity, robustness and low power losses. In addition, power quality has also been improved with Active power filtering (APF) by overcoming the drawbacks of passive filters at the line side. Hence APF becomes a promising compensator solution. This paper presents the APF for a Medium-Voltage applications using Open Loop Control Compensation

\section{References:}

[1].H. Akagi, K. Isozaki "A Hybrid Active Filter for a Three-Phase 12-Pulse Diode Rectifier Used as the Front End of a Medium-Voltage Motor Drive,” IEEE Trans. Power Electron, vol. 27, no. 1, pp. 69 - 77, 2012.

[2].M.S. Hamad, M.I. Masoud, B.W. Williams, and S. Finney, "Medium voltage 12-pulse converter: ac side compensation using a shunt active power filter in a novel front end transformer configuration," IET Trans. Power Electron, vol. 5, no. 2, pp. $1315-1323$, Sep. 2012.

[3].M. F. McGranaghan, and D.R. Mueller, 'Designing harmonic filters for adjustable-speed drives to comply with IEEE519 harmonic limits', IEEE Trans. Industry Applications, vol. 35, no. 2, pp. 312 - 318, March- April 1999.

[4].H. Akagi, "New trends in active filters for power conditioning", IEEE Trans. Industry Applications, vol. 32, no.6, pp. 1312 - 1322, Nov.- Dec. 1996. [6] A. Bhattacharya and C. Chakraborty, " A shunt active power filter with enhanced performance using ANN-based predictive and adaptive controllers," IEEE Trans. Ind. Electron., vol. 58, no. 2, pp. $421-428$, Feb. 2011

[5].M. Odavic, et al., "One-sample-period-ahead predictive current control for high-performance active shunt power filters," IET Trans. Power Electron, vol. 4, no. 4, pp. $414-423,2011$.

[6].M. S. Hamad, M. I. Masoud, B. W. Williams, "Medium-Voltage 12-pulse Converter: Output Voltage Harmonic Compensation using a Series APF," IEEE Trans. Ind. Electron., vol. 61, no. 1, pp. 43 - 52, 2014.

[7].Yi Tang, et al., "Generalized Design of High-Performance Shunt Active Power Filter With Output LCL Filter," IEEE Trans. Ind. Electron., vol. 59, no. 3, pp. 1443 - 1452, 2013.

[8].G. Cerrada et al., 'Application of a Repetitive Controller for a Three-Phase Active Power Filter', IEEE Trans. Power Electron, vol. 22, no. 1, pp. 237 - 246, Jan. 2007.

[9].S. A. Gonzalez, R.G. Retegui, R., and M. Benedetti, 'Harmonic computation technique suitable for active power Filters', IEEE Trans. Ind. Electron., vol. 54, no. 5, pp. 2791 - 2796, Oct. 2007

[10]. S. Rahmani, et al., "Experimental design of nonlinear control technique for three-phase shunt active power filters," IEEE Trans. Ind. Electron., vol. 57, no. 10, pp. 3364 - 3375, 2010.

[11]. T. Quoc-Nam, L. and Hong-Hee, "An Advanced Current Control Strategy for Three-Phase Shunt Active Power Filters," IEEE Trans. Ind. Electron., vol. 60, no. 12, pp. 5400 - 5410, 2013.

[12]. M. Aredes, H. Akagi, and E.H. Watanabe, 'Comparisons Between the p-q and p-q-r Theories in Three- Phase FourWire Systems', IEEE Trans. Power Electron, vol. 24, no. 4, pp. 924 - 933, April 2009.

[13]. S. Mariethoz, and A.C. Rufer, 'Open loop and closed loop spectral frequency active filtering', IEEE Trans. Power Electron, vol. 1, no.4, pp. $564-573,2002$.

[14]. J. Svensson and R. Ottersten, 'Shunt active filtering of vector currentcontrolled VSC at a moderate switching frequency’, IEEE Trans. Industry Applications, vol. 35, no. 5, pp. 1083 - 1090, Sept.-Oct. 1999. 